\title{
Выявляемость, коэффрициент летальности и уровень смертности от новой коронавирусной инфекции в разных субъектах Российской Федерации во время и до осенней волны эпидемии
}

Эдвард Гольдштейн (Edward Goldstein)1, ${ }^{1,}$

1. Гарвардская школа общественного здравоохранения, Бостон, США

*. Электронная почта: egoldste@hsph.harvard.edu

\section{Аннотация}

Цель: Лабораторная диагностика новой коронавирусной инфекции в совокупности с отслеживанием/карантином для контактных лиц является эфрфективным способом для уменьшения распространения новой коронавирусной инфрекции и снижения уровня соответствующей смертности. Вместе с тем, практика тестирования на новую коронавирусную инфекцию различается в разных субъектах Российской Федерации и меняется с течением времени. Важной характеристикой практики тестирования на новую коронавирусную инфекцию является выявляемость новой коронавирусной инфееции, т.е. процент выявленных случаев COVID-19 среди всех случаев заражения новой коронавирусной инфекцией в населении. В этой работе, мы оценили связь между выявляемостью, распространением и смертностью от новой коронавирусной инфекции в разных субъектах Российской Федерации. Методы: При более активном тестировании на новую коронавирусную инфекцию в населении, выявляемость новой коронавирусной инфекции увеличивается, а коэфрфициент летальности, т.е. процент летальных случаев среди всех выявленных случаев COVID-19 с известным исходом (выздоровевших + умерших) уменьшается (т.к. при более активном тестировании, выявляются больше случаев заболевания COVID-19 в легкой и 
средней форме). Поэтому, хотя выявляемость сложно оценить из данных, можно использовать коэффрициент летальности (который находится в обратной зависимости от выявляемости) для того, чтобы понять, как выявляемость связана с другими характеристиками эпидемиологического процесса.

Результаты: Коэфрфициент летальности для COVID-19 в среднем для Российской Федерации увеличился с 2.12\% в период 31/01/2020-01/10/2020 до 2.36\% в период 01/10/2020-22/11/2020; также, коэффрициент летальности для COVID-19 увеличился в 52/85 субъектах Российской Федерации в период 01/10/2020-22/11/2020 по сравнению с периодом 31/01/2020-01/10/2020. Это говорит о том, что выявляемость новой коронавирусной инфекции, в целом в Российской Федерации и в большинстве её субъектов несколько понизилась в период 01/10/2020-22/11/2020 по сравнению с периодом 31/01/2020-01/10/2020. Линейная корреляция между коэффициентом летальности в период 01/10/202022/11/2020 и уровнем смертности от новой коронавирусной инфекции на 100,000 человек в период 01/10/2020-22/11/2020 в разных субъектах Российской Федерации равна $0.54(0.37,0.67)$, а коэфрфициент корреляции Спирмена равен 0.69 (Р-значение<0.0000001). Это говорит о том, что выявляемость новой коронавирусной инфекции является одним из факторов, которые влияют на уровень смертности от COVID-19 в разных регионах России -- более высокая выявляемость способствует понижению уровня смертности от COVID-19. В ряде субъектов Российской Федерации (в частности, в г. Санкт-Петербурге), уровень смертности от COVID-19 относительно высокий, а выявляемость новой коронавирусной инфекции относительно низкая.

Заключение: Для повышения выявляемости (и понижения распространения и смертности от новой коронавирусной инфекции) следует принимать дополнительные меры для повышения уровня тестирования на новую коронавирусную инфекцию среди разных категорий лиц, включая лиц, которые хотят тестироваться на новую коронавирусную инфекцию по собственной инициативе, лиц, обращающихся за медицинской помощью с симптомами OРВИ, и контактных лиц для подтвержденных случаев COVID-19. 


\section{Введение}

Лабораторная диагностика новой коронавирусной инфекции в совокупности с отслеживанием/карантином для лиц, контактировавших с инфицированным лицом (контактных лиц) является эфрфективным способом для уменьшения распространения новой коронавирусной инфекции и снижения уровня соответствующей смертности. Например, в Исландии соответствующая диагностика и карантин/самоизоляция активно практикуются - так, используя серологические данные и данные о лабораторной (ПЦР) диагностике новой коронавирусной инфекции, исследователи оценили, что $56 \%$ всех случаев новой коронавирусной инфекции в Исландии были диагностированы [1]. Отметим также, что уровень смертности от COVID-19 в Исландии является одним из самых низких в Европе. В австралийском штате Новый Южный Уэльс, распространение новой коронавирусной инфекции в школах весной 2020-го года было очень ограниченно [2]; при этом, в школах проводилось активное тестирование на новую коронавирусную инфекцию, и лиц, контактировавших с зараженными школьниками или персоналом школы отправляли на двухнедельный карантин. В Российской Федерации тоже проводится активное тестирование на новую коронавирусную инфекцию [3], что способствует уменьшению распространения инфекции. Вместе с тем, практика тестирования на новую коронавирусную инфекцию различается в разных субъектах Российской Федерации и меняется с течением времени. Влияние различий и изменений в практике тестирования на новую коронавирусную инфекцию на распространение и уровень смертности от COVID-19 в разных субъектах Российской Федерации мало изучено.

Влияние тестирования на распространение новой коронавирусной инфеекции зависит от выявляемости новой коронавирусной инфекции (т.е. процента выявленных случаев COVID-19 среди всех случаев заражения новой коронавирусной инфекцией в населении). При более высокой выявляемости, 
лабораторно подтверждается большее количество случаев инфицирования в населении, что способствует предотвращению большего количества новых инфекций путем карантина для выявленных случаев и их контактных лиц, что уменьшает темп распространения инфекции в населении. При более активном тестировании на новую коронавирусную инфекцию в населении, выявляемость новой коронавирусной инфекции увеличивается, а коэффициент летальности, т.е. процент смертельных случаев среди всех выявленных случаев COVID-19 с известным исходом (выздоровевшие и умершие) в населении уменьшается (т.к. при более активном тестировании, выявляются больше случаев заболевания COVID-19 в легкой и средней форме). Поэтому более низкий коэффициент летальности соответствует более высокой выявляемости новой коронавирусной инфекции в разных субъектах Российской Федерации. В этой работе, мы вычислили коэффициент летальности в разных субъектах Российской Федерации в период 01/10/2020-22/11/2020 и в период 31/01/2020-01/10/2020 (для того, чтобы узнать, как выявляемость новой коронавирусной инфекции изменилась с течением времени), и оценили корреляцию между коэффрициентом летальности и уровнем смертности от новой коронавирусной инфекции в разных субъектах Российской Федерации.

Цель исследования: Оценка изменений с течением времени в выявляемости новой коронавирусной инфекцию в разных субъектах Российской Федерации. Оценка связи между выявляемостью (т.е. процентом выявленных случаев COVID-19 среди всех случаев заражения новой коронавирусной инфекцией в населении), коэффициентом летальности (процентом смертельных случаев среди всех выявленных случаев COVID-19 в населении с известным исходом (выздоровевшие + умершие)) и уровнем смертности от новой коронавирусной инфекции на 100,000 человек в разных субъектах Российской Федерации.

\section{Материалы и Методы}




\section{Данные}

Мы использовали оперативные данные о количестве выздоровевших среди выявленных случаев новой коронавирусной инфекции и количестве смертей от новой коронавирусной инфекции в разных субъектах Российской Федерации на 22/11/2020 и на 01/10/2020 [4]. Мы также использовали данные Росстата о численности населения в разных субъектах Российской Федерации на 1-го января, 2020 [5] в целях оценки уровня смертности от новой коронавирусной инфекции на 100,000 человек в разных субъектах Российской Федерации.

\section{Статистический анализ}

Мы оценили коэффрициент летальности, т.е. процент летальных случаев среди всех выявленных случаев COVID-19 с известным исходом (выздоровевших + умерших) в разных субъектах Российской Федерации в период 01/10/202022/11/2020 и в период 31/01/2020-01/10/2020. Мы также оценили корреляцию между коэффрициентом летальности и уровнем смертности от новой коронавирусной инфекции на 100,000 человек в разных субъектах Российской Федерации в период 01/10/2020-22/11/2020.

\section{Результаты}

На Рис. 1 изображены коэффрициенты летальности, т.е. процент летальных случаев среди всех выявленных случаев COVID-19 с известным исходом (выздоровевших + умерших) в разных субъектах Российской Федерации в периоды 01/10/2020-22/11/2020 и 31/01/2020-01/10/2020. Коэффициент летальности для COVID-19 в среднем по Российской Федерации увеличился с $2.12 \%$ в период 31/01/2020-01/10/2020 до 2.36\% в период 01/10/2020-22/11/2020; также, коэффрициент летальности для COVID-19 увеличился в 52/85 субъектах Российской Федерации в период 01/10/2020-22/11/2020 по сравнению с 
периодом 31/01/2020-01/10/2020, что говорит о понижении выявляемости новой коронавирусной инфекции относительно периода 31/01/2020-01/10/2020.

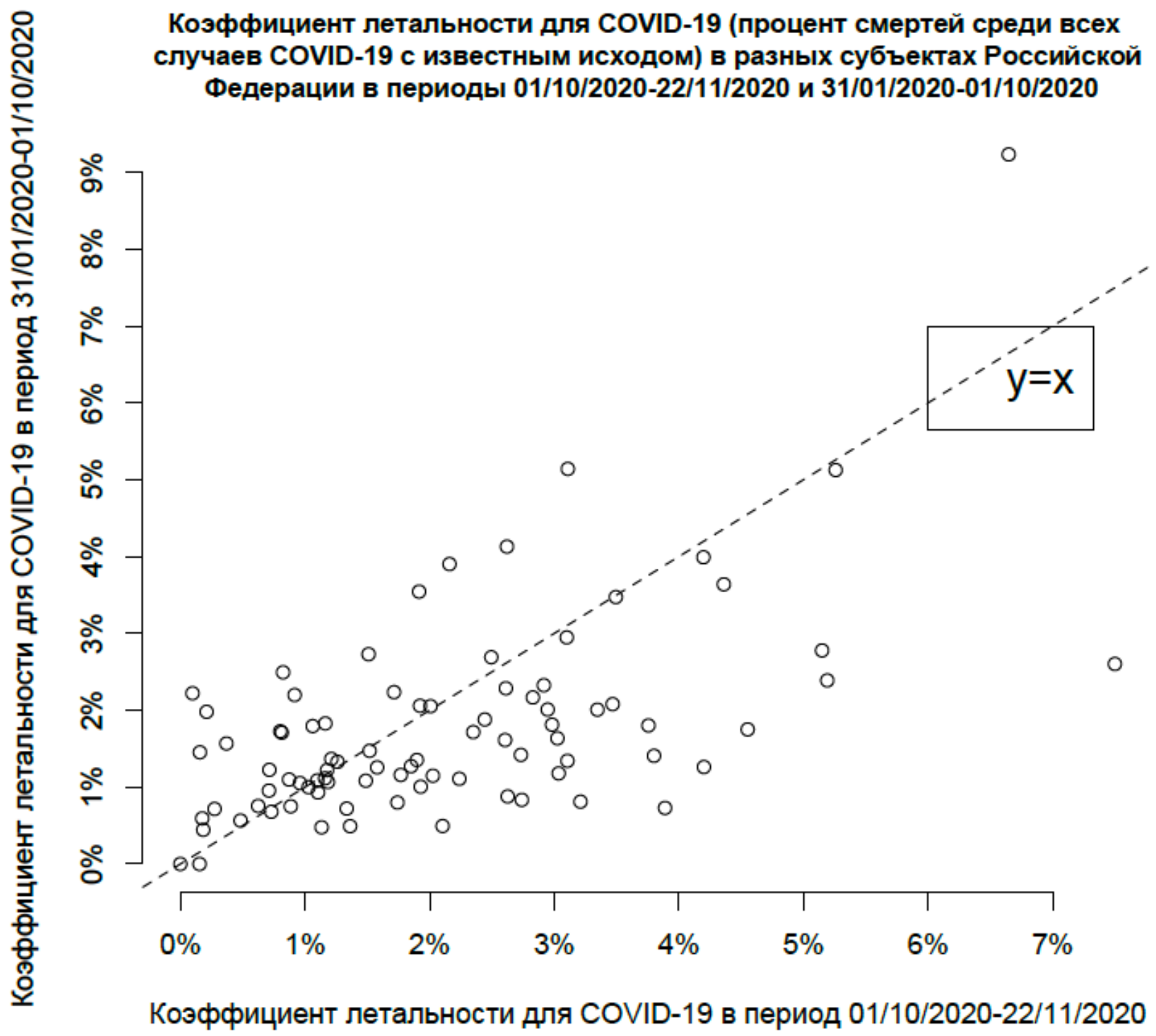

Рис. 1: Коэффрициенты летальности, т.е. процент летальных случаев среди всех выявленных случаев COVID-19 с известным исходом (выздоровевших + умерших) в разных субъектах Российской Федерации в периоды 01/10/202022/11/2020 и 31/01/2020-01/10/2020. 
На Рис. 2 изображены коэффрициенты летальности и уровни смертности от новой коронавирусной инфекции на 100,000 человек в разных субъектах Российской Федерации в период 01/10/2020-22/11/2020. Линейная корреляция между коэфффициентом летальности в период 01/10/2020-22/11/2020 и уровнем смертности от COVID-19 на 100,000 человек в период 01/10/2020-22/11/2020 в разных субъектах Российской Федерации равна 0.54 (0.37,0.67), а коэффициент корреляции Спирмена равен 0.69 (Р-значение <0.0000001). Это говорит о том, что выявляемость новой коронавирусной инфекции является одним из фракторов, которые влияют на уровень смертности от новой коронавирусной инфекции в разных регионах России -- более высокая выявляемость способствует понижению уровня смертности от новой коронавирусной инфекции. В ряде субъектов Российской Федерации, уровень смертности от новой коронавирусной инфекции относительно высокий, а выявляемость новой коронавирусной инфекции относительно низкая (коэффрициент летальности относительно высокий, Рис. 2). В частности, в г. Санкт-Петербурге, уровень смертности от новой коронавирусной инфекции, как в период 01/10/202022/11/2020, так и в период 31/01/2020-01/10/2020 является самым высоким в Российской Федерации, а выявляемость новой коронаварусной инфекции одна из самых низких в Российской Федерации. При этом, выявляемость новой коронавирусной инфрекции в г. Санкт-Петербурге несколько увеличилась со временем (коэфффициент летальности уменьшился с 9.23\% в период 31/01/202001/10/2020 до 6.65\% в период 01/10/2020-22/11/2020, Рис. 1). 


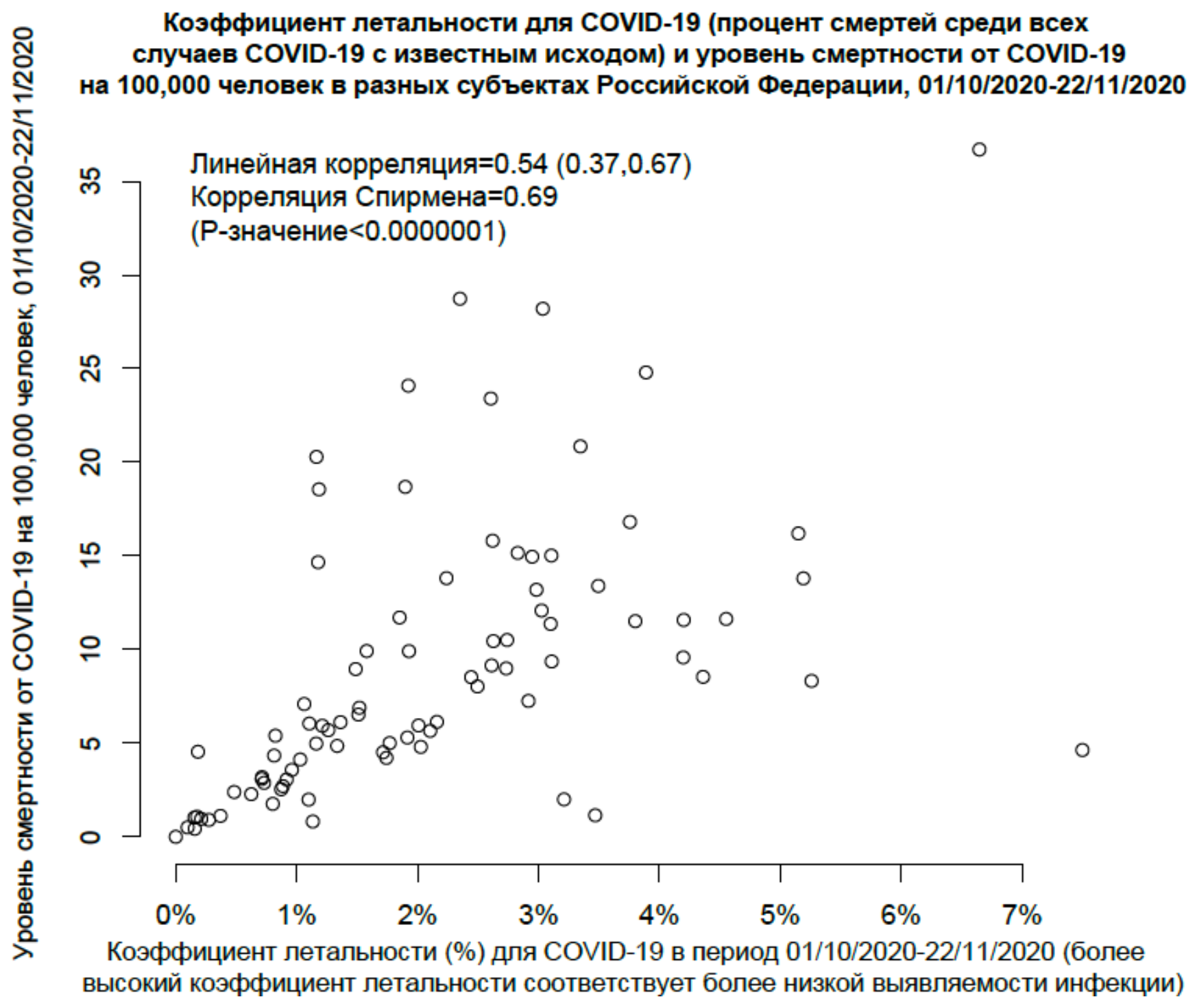

Рис. 2: Коэффициенты летальности, т.е. процент летальных случаев среди всех выявленных случаев COVID-19 с известным исходом (выздоровевших + умерших) и уровни смертности от новой коронавирусной инфекции на 100,000 человек в разных субъектах Российской Федерации в период 01/10/2020$22 / 11 / 2020$.

\section{Выводы}


Активное тестирование на новую коронавирусную инфекцию с последующим карантином для инфицированных и контактных лиц способствует уменьшению распространения новой коронавирусной инфекции [6] и снижению уровня соответствующей смертности. Высокий уровень тестирования и выявления новой коронавирусной инфекцию наблюдается в ряде стран [1,7]. Это приводит к высокому уровню выявляемости новой коронавирусной инфекции (т.е. проценту выявленных случаев COVID-19 среди всех случаев заражения новой коронавирусной инфекцией в населении) - так, в Исландии выявляемость новой коронавирусной инфрекции оценена в 56\% [1]. В Российской Федерации тоже проводится активное тестирование на новую коронавирусную инфекцию [3]. Вместе с тем, критерии и практика тестирования на новую коронавирусную инфекцию различаются в разных субъектах Российской Федерации и меняются с течением времени. Например, в ряде субъектов Российской Федерации практикуется тестирование на новую коронавирусную инфекцию для всех людей с респираторными симптомами, которые обращаются за медицинской помощью [8,9]; в ряде других субъектов Российской Федерации, в амбулаторных условиях до 27/10/2020 [3] тестировали только определённые категории лиц (людей старше 65-и лет, медицинских работников, и др.) [10,11]. Влияние различий и изменений в практике тестирования на новую коронавирусную инфекцию на уровень заболеваемости и смертности от новой коронавирусной инфекции в разных субъектах Российской Федерации мало изучено.

В этой работе, используя оперативные данные [4] о количестве выздоровевших и умерших от новой коронавирусной инфекции, мы нашли, что выявляемость новой коронавирусной инфекции, в целом по Российской Федерации и в большинстве её субъектов несколько понизилась в период 01/10/202022/11/2020 по сравнению с периодом 31/01/2020-01/10/2020. Это говорит о том, то уровень тестирования на новую коронавирусную инфекцию, в целом по Российской Федерации и в большинстве её субъектов, не вырос во время осенней волны эпидемии по сравнению с весенней/летней волной эпидемии. 
Также, мы нашли, что выявляемость новой коронавирусной инфекции является одним из фракторов, которые влияют на уровень смертности от новой коронавирусной инфекции в разных регионах России -- более высокая выявляемость способствует понижению уровня смертности от новой коронавирусной инфекции. Для повышения выявляемости следует принимать дополнительные меры для повышения уровня тестирования на новую коронавирусную инфекцию среди разных категорий лиц, включая лиц, которые хотят тестироваться на новую коронавирусную инфекцию по собственной инициативе, лиц, обращающихся за медицинской помощью с симптомами OРBИ, и контактных лиц для подтвержденных случаев COVID-19. Такие меры в совокупности с карантином для инфицированных и контактных лиц способствует понижению уровня заболеваемости и смертности от новой коронавирусной инфекции.

\section{Список литературы}

[1] Gudbjartsson DF, Norddahl GL, Melsted P, et al. Humoral Immune Response to SARS-CoV-2 in Iceland. New England Journal of Medicine 2020. https://www.nejm.org/doi/full/10.1056/NEJMoa2026116

[2] Macartney K, Quinn HE, Pillsbury AJ, et al. Transmission of SARS-CoV-2 in Australian educational settings: a prospective cohort study. Lancet Child \& Adolescent Health, 2020. Available from:

https://www.thelancet.com/journals/lanchi/article/PIIS2352-4642(20)30251-0/fulltext

[3] Министерство Здравоохранения Российской Федерации. ПРОФИЛАКТИКА, ДИАГНОСТИКА И ЛЕЧЕНИЕ НОВОЙ КОРОНАВИРУСНОЙ ИНФЕКЦИИ (COVID19). Версия 9 (26.10.2020). https://static- 
0.minzdrav.gov.ru/system/attachments/attaches/000/052/550/original/\%D0\%9C\%D0\% A0 COVID-19 \%28v9\%29.pdf?1603788097

[4] Стопкоронавирус.РФ. Оперативные данные. 2020. https://стопкоронавирус.pdp/

[5] Федеральная служба государственной статистики (Росстат). Численность постоянного населения на 1 января. 2020. https://showdata.gks.ru/report/278928/

[6] World Health Organization. Contact tracing in the context of COVID-19. 2020. https://www.who.int/publications/i/item/contact-tracing-in-the-context-of-covid-19

[7] Park YJ, Choe YJ, Park O, et al. Contact Tracing during Coronavirus Disease Outbreak, South Korea, 2020. Emerging Infectious Diseases 2020; 26(10).

[8] Департамент здравоохранения города Москвы. Диагностика COVID-19 в Москве. Молекулярная диагностика. 8 июня 2020 г. https://niioz.ru/covid19/info/profilaktika-i-lechenie/Kak\%20zashchitit\%27\%20sem\%27yu\%20i\%20dom/

[9] МИНИСТЕРСТВО ЗДРАВООХРАНЕНИЯ РЕСПУБЛИКИ БАШКОРТОСТАН. О совершенствовании лабораторной диагностики на COVID-19 в Республике Башкортостан (с изменениями на 19 июня 2020 года). http://docs.cntd.ru/document/570822549

[10] МИНИСТЕРСТВО ЗДРАВООХРАНЕНИЯ ЧЕЛЯБИНСКОЙ ОБЛАСТИ. О группах пациентов, подлежащих обследованию на коронавирусную инфрекцию (COVID-19) (с изменениями на 14 сентября 2020 года). http://docs.cntd.ru/document/570812390

[11] МИНИСТЕРСТВО ЗДРАВООХРАНЕНИЯ СВЕРДЛОВСКОЙ ОБЛАСТИ. О внесении изменений в Приказ Министерства здравоохранения Свердловской 
области от 27.03.2020 N 491-п "Об организации лабораторной диагностики новой коронавирусной инфекции в лабораториях учреждений здравоохранения Свердловской области". ПРИКАЗ от 8 июня 2020 года N 1021-п.

http://docs.cntd.ru/document/570823262 